\title{
Transfer Function Based Band Selection in Frequency Domain Time Series Regression
}

\author{
Skye Griffith ${ }^{1}$, Glen Takahara ${ }^{1}$, Wesley S. Burr ${ }^{2}$ \\ ${ }^{1}$ Department of Mathematics \& Statistics, Queen's University \\ 99 University Avenue, Kingston, Ontario, Canada \\ skye.griffith@queensu.ca; takahara@queensu.ca, \\ ${ }^{2}$ Department of Mathematics, Trent University \\ 1600 West Bank Drive, Peterborough, Ontario, Canada \\ wesleyburr@trentu.ca
}

\section{Extended Abstract}

This work provides a method of time series regression that is applicable to a general class of stationary time series processes containing harmonic components. Time series regression provides a model for a response time series as a function of one or more predictor time series, and in addition, showcases the evolution of relationships between predictors and response over time. Unfortunately, standard time-domain regression models don't account for any temporal factors that may be present, operating under an assumption of independence that is commonly unsatisfied by time series data. A useful alternative to standard regression is to implement a linear filter model, which convolves linear filter coefficients with a predictor time series and then adds an error term to account for noise. The interpretation of filter coefficients is unclear, however, as their meaning is not necessarily analogous to that of standard regression coefficients [1]. Analysing the model instead via complex regression in the frequency domain, by way of a transfer function, provides insight into relationships between signals present in predictor and response series. Identifying the distribution of the modulus and phase of a transfer function allows for further interpretation of the model.

In the multitaper framework for spectrum estimation, the transfer function is expressed in terms of the eigencoefficients of the predictor and response signals. The multitaper method controls broadband bias, is known to be an approximately maximum likelihood estimator of the spectrum, and the eigenspectra (the squared modulii of the eigencoefficients) are a collection of uncorrelated direct spectrum estimators [2]. In the multitaper framework, the transfer function estimator at each frequency is the complex regression coefficient obtained by regressing the response eigencoefficients onto the predictor eigencoefficients. While the limiting distributions of the eigencoefficients and the transfer function at each frequency are known in the case that the response and predictor series are white noise processes, less is known about the distributions when the underlying processes contain harmonic elements embedded in more general stationary noise.

In the context of time series regression, we wish to relate structural temporal components in the response to those in the predictor. In the frequency domain this entails band selection to isolate those frequency bands in which those structural components live in both the response and predictor time series. In this talk we explore the distributional behaviour of the multitaper transfer function estimator in bands where there is common structure in the response and predictor versus bands where there is no such common structure. We discuss the joint distribution of the complex eigencoefficients, the multitaper transfer function estimator, and the modulus and phase of the transfer function. We investigate the use of the transfer function as a detector for frequency bands that contain line components in both response and predictor, and the development of statistical tests based on the differential distributional properties of the transfer function "in-band" versus "out-of-band".

\section{References}

[1] Gasparrini, Antonio, Fabian Scheipl, Ben Armstrong, and Michael G. Kenward. "A penalized framework for distributed lag non-linear models," Biometrics, vol. 73, no. 3, pp. 938-948, 2017.

[2] David J. Thomson. "Spectrum estimation and harmonic analysis," Proceedings of the IEEE, vol. 70, no. 9, pp. 10551096, 1982 